\title{
Fusion of Intracardiac Ultrasound with 3D Cardiac C-arm CT from Animal Data for Electrophysiology
}

\author{
Matthias John ${ }^{1}$, Yiyong Sun $^{2}$, Samuel Kadoury ${ }^{2}$, Wolfgang Wein ${ }^{2}$, \\ Yong $\mathrm{Li}^{2}$, Jeff Resnick ${ }^{3}$, Gerry Plambeck ${ }^{3}$, Ann Dempsey, \\ Amin Al-Ahmad ${ }^{4}$, Rebecca Fahrig ${ }^{4}$, Frank Sauer ${ }^{2}$ \\ ${ }^{1}$ Siemens Medical Solutions, Erlangen, Germany \\ ${ }^{2}$ Siemens Corporate Research, Princeton, NJ, USA \\ ${ }^{3}$ Siemens Medical Solutions, Mountain View, CA, USA \\ ${ }^{4}$ Stanford University Medical School, Stanford, CA, USA \\ Email: matthias.mj.john@siemens.com
}

\begin{abstract}
Summary. In Electrophysiology catheter ablation is an established treatment for cardiac arrhythmias. Nevertheless, complicated ablation procedures such as Pulmonary vein isolation for atrial fibrillation treatment are difficult to learn. This can be improved by a better integration of the image modalities available today and in the future Electrophysiology lab. In this paper, we present a method to register ultrasound images from an Intracardiac Echo catheter and 3D cardiac C-arm CT images, which can both be obtained during the intervention. This is the first step required to display the Intracardiac Echo images relative to the complex anatomy of the left atrium.
\end{abstract}

\section{Introduction}

Atrial fibrillation is the most common heart arrhythmia and the major cause of stroke. Over 2 million people are affected in the U.S. alone. Atrial fibrillation can be treated using a Pulmonary vein isolation procedure. Here the Pulmonary veins are electrically isolated from the left atrium by a radio frequency ablation catheter, with image guidance provided today mainly by x-ray fluoroscopy. Only highly trained Electrophysiologists are able to perform the procedure, because of the complex and patient specific anatomy of the left atrium. Cardiac CT and MR can deliver high resolution 3D images of the individual heart anatomy. In the future, this pre-interventional imaging could be replaced by a rotational $\mathrm{C}$-arm technique, that is able to produce such images immediately before or during the intervention [1]. An imaging modality that is already used today in many EP labs is Intracardiac Echo (ICE) - a steerable catheter that contains an ultrasound transducer in its tip. By placing the catheter tip into the right atrium the physician is able to image the whole left atrium and some neighboring structures in real time. Therefore it has become an excellent tool for visualization of anatomical structures and instruments, and to monitor critical events.

The combination of 3D cardiac rotational C-arm imaging with ICE and a future integration in the EP lab could help the Electrophysiologists to guide ICE and the ablation catheter. It could improve the learning curve for the use of ICE and therefore the whole Pulmonary vein isolation procedure. 


\section{State of the art and advances by the presented contribution}

Several approaches have been suggested for the registration of ultrasound with MR and conventional CT, e.g. in [2] for kidney images from ultrasound and CT. There the ultrasound images are preprocessed by reducing speckle noise and shadows. In [3] a similarity measure is presented that consists of skin surface clamping (not necessary for ICE), edge correlation and Mutual Information. CT intensities, gradients and edge features are used for the computation.

Also for Electrophysiological applications ultrasound was fused with CT data. In [4] a registration between conventional cardiac CT data and Intracardiac Echo is described. A point-to-surface registration, by first extracting surface point sets of the left atrium from the ICE images, is used.

As far as we know, our results are the first on fusing the two image modalities Intracardiac Echo and cardiac C-arm CT.

\section{Methods}

\subsection{System}

In our system 3D images are acquired on a Angiographic C-arm system (AXIOM Artis, Siemens Medical Solutions). To image the left atrium of a patient we acquire images during 4 consecutive rotational $190^{\circ} \mathrm{C}$-arm runs. Therefore we get enough images to reconstruct a 3D image of one cardiac phase. The images are reconstructed and processed on a PC workstation. The left atrium and other heart structures can be segmented using dedicated software.

The images acquired by the ICE catheter (AcuNav, Siemens Medical Solutions) are transferred via a frame grabber card into the PC. To track the position of the ICE catheter tip we used a magnetic tracking system (Microbird, Ascension). Its position sensor has been integrated in the same tubing with the ultrasound transducer. The transmitter is installed under the table of the C-arm system, such that an ICE catheter above the table can be tracked during an intervention.

During ICE imaging we record the ECG signal of the patient, and track the position of the ICE position sensor and a position sensor at the patient's chest (for correcting respiratory motion) synchronously.

\subsection{Experimental animal data}

In an animal experiment a 3D cardiac C-arm CT data set from a pig was taken. We additionally took various Intracardiac Echo image sequences from the pig's heart. The catheter was inserted by the physician into the right atrium of the pig. All image sequences were taken from this position by rotating and slightly moving the catheter tip. As mentioned above we also recorded the ECG signal and the coordinates of the two position sensors at catheter tip and chest. Based on these images we performed the following preprocessing and registration procedure offline. 
Figure 1. Preprocessing of a pig heart cardiac C-arm CT image: original image (left), gradient magnitude image (middle), and after applying a mask with a threshold of 67 (right).
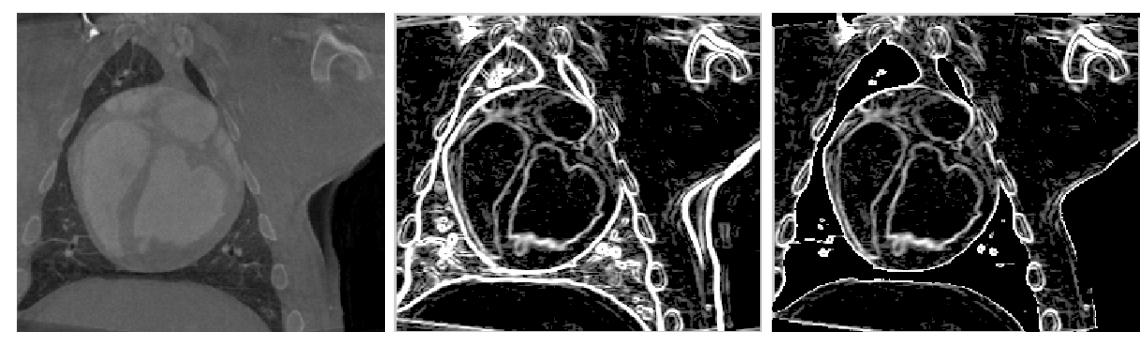

\subsection{Motion gating for ICE images}

The main difficulty of a good registration is the cardiac motion from the beating heart and the motion due to respiration in the ICE images. Therefore we need a good preselection of useful ICE images.

If we ignore patient movement we can observe the cyclic respiration motion in the graph of the vertical dimension of the $3 \mathrm{D}$ position sensor at the chest. This graph has regular peaks separated by long plateaus. The plateaus are of nearly constant height. We compute for every image the variation of this image and its previous images for a fixed time frame. For images with a low variation we can assume that these images were taken in a respiratory phase corresponding to a plateau. So we select those 'low variation' images from the whole sequence.

To compensate for cardiac motion we further select those images with a fixed time distance to the previous R-wave in the ECG signal.

\subsection{Registration}

For an initial registration the user has to select a point in the $3 \mathrm{D}$ data set that is close to the position of the catheter tip of the ultrasound image sequence. This gives an initial translation. For an initial rotation we assume that the tracking device is installed under the $\mathrm{C}$-arm table in a fixed and given direction.

This initial registration is followed by an automatic local optimization step to find a good rigid body transformation. First we preprocess the C-arm CT data. We extract the magnitudes of the local gradients by applying a 3D Sobel filter [5]. Because there are many regions outside the heart with strong gradients that can worsen the registration quality, we apply a mask based on the grey values of the original volume to focus on the edges representing the heart walls (see Fig. 1). The ultrasound images are downsized by a factor of 4 in each dimension to improve the runtime.

We optimize the transformation according to the following similarity measure: We re-slice the C-arm CT gradient magnitude data in the ultrasound image planes using tri-linear interpolation. Now the similarity measure is computed using a Normalized cross-correlation [5]: 


$$
\text { similarity }_{T}=\sum_{i=1}^{n} \operatorname{NCC}\left(U S_{i}, C T_{T, i}\right) \text {, }
$$

where $U S_{i}$ is the $i$ th ICE image from the gated sequence of $n$ images. $C T_{T, i}$ is the resliced plane from the cardiac C-arm CT gradient magnitude data corresponding to $U S_{i}$ and a given transformation $T$. Observe that we take the measure only for those pixels located inside the fan shape of the ultrasound image.

The optimization is done using a best neighbor method. For an initial step size all transformation parameters are changed in turn with the step size and the resulting value of the similarity measure is computed. The change with the best improvement is taken and the step is repeated until there is no further improvement. Then the step size is decreased and the whole procedure starts again. We use an initial step size of $5.5 \mathrm{~mm}$ and 5.5 degrees. Both are reduced by a factor of 2 repeatedly until we reach a step size of $0.1 \mathrm{~mm}$ or 0.1 degree.

\section{Results}

The registration was done offline with the cardiac C-arm CT and ICE data obtained from the animal experiment. A visual comparison of the registration can be obtained by aligning the ICE images side by side with their corresponding cardiac C-arm CT cut planes (see Fig. 2).

For a quantitative validation of the registration results we compared segmentations of cardiac chambers. For the 3D segmentation of the C-arm CT data set we used a semi-automatic tool developed for cardiac CT data. The segmentation of the ICE data was done manually by an expert.

We generated registrations and segmentations of 29 pairs of ICE images and their corresponding $\mathrm{C}$-arm $\mathrm{CT}$ cut planes. For visual assessment we compared the contour of the ultrasound segmentation and the registered C-arm CT segmentation contour (see Fig. 3). For quantitative assessment we computed the shortest distance from each contour pixel of the $\mathrm{C}$-arm $\mathrm{CT}$ segmentation to the registered ultrasound contour. The mean error was $3.14 \pm 3.13 \mathrm{~mm}$.

The whole registration procedure implemented in $\mathrm{C}++$ took less than a minute on a system with an Intel P4 processor with $2.8 \mathrm{GHz}$ and $2 \mathrm{~GB}$ DDR memory.

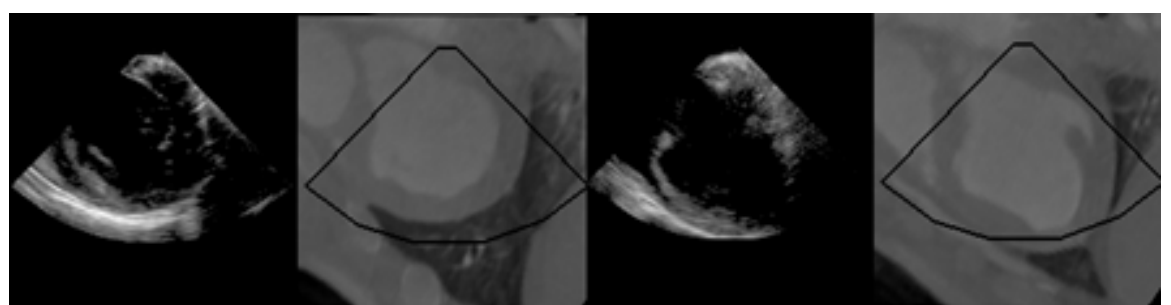

Figure 2. Registration using a sequence of 12 gated ICE images. 
Figure 3. Contour of a cardiac chamber manually segmented from ultrasound (left). The contour of the same chamber segmented from the cardiac C-arm CT image and projected to the registered ultrasound image (middle). A fusion of an ICE image with segmented cardiac chambers from the 3D cardiac C-arm CT image (right).
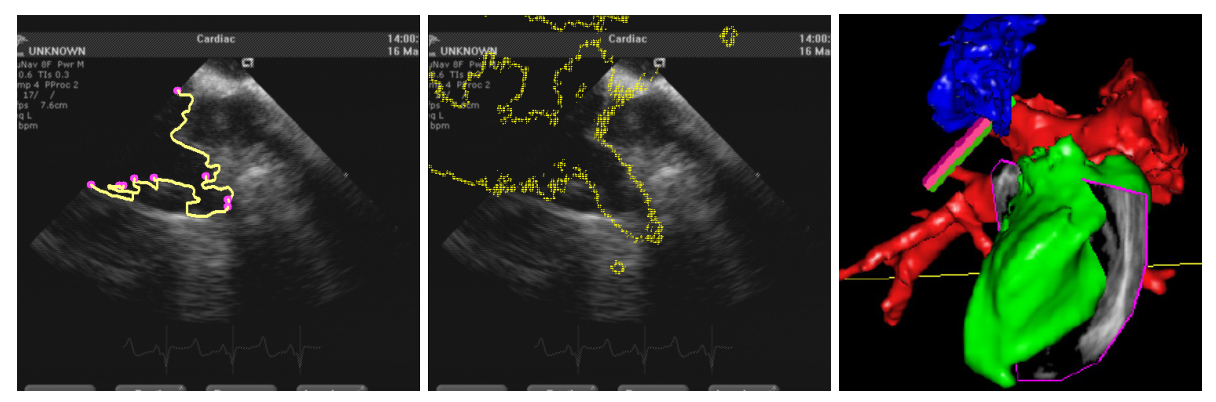

\section{Discussion}

The results show a good alignment of ICE images and their registered cardiac C-arm CT planes. Nevertheless, the quantitative analysis of registration accuracy shows some variation. The question is, whether this is related to the registration algorithm. A non-negligible additional source of registration and fusion errors that we currently ignore might be patient movement. Furthermore the registration algorithm is based on grey values, whereas the validation of these results is based on segmentations. Some segmented anatomical details and their contour lines might be different in both image modalities. In particular, a precise manual segmentation of the ultrasound images is difficult to achieve.

In the future our proposed methods can be used to build a system that makes it easy to integrate and fuse 3D cardiac rotational C-arm imaging and Intracardiac Echo in the EP suite. Both image modalities will be available during the EP procedure, in contrast with MR and CT which are acquired pre-intervention. A further step could be the fusion with Electro-anatomical mapping data. We believe that these image integrations make it much easier for physicians to learn and perform complex EP ablation procedures.

\section{References}

1. Lauritsch G, et al: Towards Cardiac C-Arm Computed Tomography. IEEE Trans Med Imaging 25(7): 922-934, 2006.

2. Leroy A, et al.: Rigid Registration of Freehand 3D Ultrasound and CT-Scan Kidney Images. Procs MICCAI 2004: 837-844, 2004.

3. Wein W, Roper B, Navab N: Automatic registration and fusion of ultrasound with CT for radiotherapy. Procs MICCAI 2005: 303-311, 2005.

4. Zhong H, Kanade T, Schwartzman D: "Virtual Touch": An Efficient Registration Method for Catheter Navigation in Left Atrium. Procs MICCAI 2006:437-444, 2006.

5. Gonzalez RC and Woods RE: Digital Image Processing. Prentice Hall, 2002. 